\title{
Barriers To Optimal Index Testing for Improved HIV Positivity Yield in Lusaka Urban District of Zambia - A Research Protocol
}

\author{
Cibangu Katamba \\ HIV/TB Mentorship Program, Lusaka Provincial Health Office, Lusaka, Zambia
}

\begin{abstract}
As the proportions of people living with HIV who do not know their HIV infection status decrease, reaching the last mile of those who are asymptomatic and not in contact with the health care system becomes a critical challenge. This project will use an explanatory sequential mixed-methods study design. It will be conducted in three high-volume health facilities in Matero sub-district 3 of Lusaka district in Zambia over a period of one year. The specific objectives are: to understand the perceived facilitators and barriers to HIV partner testing from the perspective of the health-care provider; to propose interventions necessary for improved HIV case finding; and to reach high risk but hard to reach populations in HIV programs such as middle-aged men and adolescent girls and young women $(A G Y W)$. The permission to conduct this study will be obtained from the Lusaka provincial health office before its commencement. Ethical clearance will be sought and obtained from the ERES Converge research ethical committee. The authority to conduct research will be sought from the National Health Research Authority. The expected main findings following the address of the identified challenges are: increased number of recipients of care indexed, increased index contact elicitation ration from 1:1 to at least 1:2, improved rate of contacts tested on time, improved HIV case finding through index testing, and overall, increased HIV index testing positivity rate from below 25\% (baseline) to above $40 \%$ (at the end of the project).
\end{abstract}

Keywords: Barriers, HIV, index testing, Lusaka, positivity yield.

\section{Introduction}

There were an estimated 36.9 million people living with HIV in 2018, based on the UNAIDS Global AIDS Update report [1]. Recently, marked progress on HIV test and treat strategy has been achieved by countries' commitment to achieving the 90-90-90 targets by 2020 [1]. As of December 2017, three out of every four people living with HIV knew their HIV status globally; $90 \%$ of HIV-infected individuals are expected to know their HIV status by the year 2020 [1].

According to ZAMPHIA 2016 fact sheet [2], only $67.3 \%$ of people living with HIV (ages 15 - 49) knew their HIV status. In 2017, Zambia had 1.1 million people living with HIV and 48,000 new HIV infections [3]. Without HIV testing services interventions targeted to key populations, including sexual partners of index clients infected with HIV, it will be hard to end the HIV epidemic by 2030 [4].

Many studies have shown that index partner testing has the potential to increase HIV testing services (HTS) uptake; identify and diagnose HIV-infected partners (yield ranging from 35\% to $62 \%$ without reported intimate partner violence (IPV) [5].

However, they are barriers to effective HIV index testing. Some reviewed challenges pertaining to both male and female index clients noted are difficulties notifying past or casual partners [6]. Disease symptoms are a motivating factor for HIV testing for men as well as women. Studies have shown that gender determinants 
such as tolerant attitudes about intimate partner violence and unequal power dynamics within relationships had noticeable effects for men and women on deciding to get tested for HIV [7].

Gender-specific barriers for female index clients to successful referral include the following: women face gender roles and inequalities in relationships such as lack of education, lack of resources or ability to access services, fear of abandonment, violence or other abuse associated with partner notification $[6,8$, 9]. They may need additional support to overcome challenges in the partner notification process. Antenatal care exposure makes women more likely to get tested for HIV.

For men, especially those in Sub-Saharan Africa, the following challenges to HIV testing and disclosure were noted: stigma, gender, and social roles prescribing that men should be healthy, strong, and dominant $[10,11]$. Studies have shown that men, compared to women, underestimate their level of risk of HIV infection [12]. However, once men have tested, they may be more likely to disclose their HIV status [12]. Some men believe that their role of breadwinner for the family protects them from rejection when disclosing their HIV-positive status [13].

Rational: Effective approaches to HIV testing are needed to reach undiagnosed people and link them to HIV care and treatment as part of the UNAIDS 90-90-90 goals. Understanding barriers to index testing is crucial for planning appropriate interventions to improve HIV testing yield and to provide appropriate care for both index clients and their partners.

Generating such evidence requires a combination of quantitative and qualitative research methods.

In this context, we will aim to assess the barriers to optimal index testing for improved HIV testing yield in the Lusaka urban district of Zambia.

Significance: The cornerstone for achieving the UNAIDS 90-90-90 targets by the year 2020 begins with people living with HIV (PLHIV) knowing their status. As the proportions of those living with HIV who do not know their HIV infection status decrease, reaching the last mile of those who are asymptomatic and not in contact with the health care system becomes a critical challenge. Therefore, reaching the first 90 will require effective and efficient HIV testing approaches. The number of people living with HIV who know their HIV status and those who receive antiretroviral therapy could increase by expanding index testing services. This will result in the reduction of the number of people who can transmit the virus and subsequently in reduced new HIV infections.

The goal of index testing is to break the chain of HIV transmission by offering HTS to persons who have been exposed to HIV.

The specific objectives are 1) to understand the perceived facilitators and barriers to HIV partner testing from the perspective of the health-care provider; 2) to propose interventions necessary for improved HIV case finding, and 3) to reach high risk but hard to reach populations in HIV programs such as middle-aged men, and adolescent girls and young women (AGYW).

The permission to conduct this study will be obtained from the Lusaka provincial health office before its commencement. Ethical clearance will be sought and obtained from the ERES Converge research ethical committee. Authority to conduct research will be sought from the National Health Research Authority.

\section{Methodology}

\section{Study Design}

An explanatory sequential mixed-methods study design will be used. In the quantitative phase, a retrospective study will be looking at index registers of clients who tested HIV positive and were elicited for index testing between July and September 2019. This will be conducted in three high-volume health facilities in Matero sub-district 3 of Lusaka district in Zambia. The overview results of the study, which will look at the effectiveness of HIV index testing, will be described. The analysis will examine index clients' identification, elicitations 
of index contacts, and testing of index contacts. The main quantitative outcome of interest for this analysis will be a success of index testing to improve yield for HTS among females versus male and between ages among index clients; and secondly, ART initiation for positive index contacts. This will be followed by a qualitative phase, one-to-one interview to explore index testing providers' views on barriers and other experiences arising during the process of HIV index testing. An interview guide will be used for consistency of information to be collected. The study will be concluded by a six to nine months optimized index testing through partner notification services, following on-the-job mentorship (addressing identified barriers) of index testing providers.

\section{Study Setting}

Lusaka is Zambia's highest HIV prevalence province, with $16.1 \%$ of the people living with HIV (ZAMPHIA 2016). The study facilities will include Matero first-level hospital, Matero Main clinic, George health Centre (in Matero Subdistrict 3 of Lusaka Urban district, Zambia).

\section{Study Population}

\section{Quantitative}

The study population will comprise all index clients (men and women at the study facilities who had been diagnosed with HIV and elicited for HIV index contact testing during the study period. This retrospective phase will use a total sample enumeration technique.

\section{Qualitative}

HIV index testing providers involved in patient care and management (Index testing champions, nurses, medical officers, counsellors, and community liaison officers) will be interviewed. Key informants will include medical officers, health systems strengthening nurses, index community liaison officers, and index testing counselors). A saturation of findings will be used to guide the sample size.

\section{Eligibility Criteria}

Participants in the final phase of the study are estimated to be 370 or more index cases (men and women at the study facilities who are newly diagnosed with HIV or newly enrolled in care, through either VCT or PICT) and their sexual contacts (sexual partners of index clients who have been elicited and offered HIV index testing services). The study participants will include:

- Newly diagnosed HIV positive or newly enrolled in HIV care/ART (for less than 6 months);

- Aged 18 years and above;

- Had a sexual partner currently or in the past 12 months.

- Eligibility criteria for enrollment as a sexual partner will be that the partner is 18 years of age or older.

\section{Exclusion Criteria}

- Known HIV clients on ART for 6 months or more.

- ART LTFU (Lost to follow up) and those with a high viral load of more than 1000 copies/ml.

- Age below 18 years.

- Sexual contact more than 12 months ago.

\section{Sampling}

The sample size for the study is calculated to answer the main study objectives, which is the improved index testing yield among participants. The sample size calculation for the original research question is based on the assumption that index clients would list an average of one sexual contact and that $51 \%$ of sexual partners would accept HIV index testing, as seen in a study conducted in the hospital setting in Malawi [14] and replicated in Tanzania[15]. The determination of the sample size is done through the approach based on precision rate and confidence level.

Using the Zambia Ministry of Health $(\mathrm{MOH})$ HIV program target indicators, it is projected that optimized HIV testing services will yield a positivity rate of $26 \%$. 
Based on these assumptions, an effective sample size of 296 index contacts will be needed to detect a similar rate of testing among sexual contacts with $80 \%$ power $(\alpha=0 \cdot 05$, two-sided test).

The sample size formula is:

$\mathrm{n}=1.96^{2} \times \mathrm{p} \times(1-\mathrm{p}) / \mathrm{e}^{2}=1.96^{2} \times 0.26 \times(1$ $-0.26) /(0.05)^{2}=296$.

Anticipating a non-response or dropout a percentage of $20 \%$, the final sample size is calculated as follows: $296 / 1-0.2=370$.

\section{Study Procedures}

\section{Review of data}

Retrospective review of index registers of clients who tested HIV positive and were elicited for index testing between July and September 2019.

\section{Qualitative study}

Participants in the qualitative component of the study will be selected on a convenience basis from the index testing services providers. The index providers will be selected in equal numbers from male and female index testing services providers who have been providing index testing for more than six months. Index providers will be selected by the principal investigator who is trained in qualitative and quantitative studies for participation in a face-toface interview on a convenience basis. A rough quota will be given to each facility, balancing out male and female participants. Those providers who will be invited to participate and agree and consent will be interviewed. This procedure intends to have a large enough sample size to uncover a variety of opinions but to limit the sample size at the point of saturation (occurring when adding more participants to the study does not result in obtaining additional perspective or information).

\section{Intervention study}

Potentially eligible men and women (diagnosed with HIV through VCT or PICT at the study facilities) will be offered HIV index testing services as per national guidelines. HIV- trained index testing counselors (study staff) will screen potential candidates for study eligibility using eligibility criteria for enrollment as index clients.

Written informed consent will be obtained from interested and eligible clients before enrolling them as index cases.

Index cases will elicit names, and contact information (phone number and physical address) for sexual partners to study staff, who then will enter the elicited contacts in index registers. Index clients also will decide how the partner will be contacted for HIV index testing services.

Index clients will be encouraged to elicit as many partners as they can, indicating the type (married, unmarried, casual partner, boyfriend, or girlfriend), duration, and status (past or current) of the relationship for each partner.

During partner elicitation, the index client will be asked questions designed to identify sexual contacts to whom disclosure or the referral to HIV index testing might cause a risk of intimate partner violence (IPV). Any sexual partners the index client feels might react with violence will be excluded from index testing services.

Index cases would choose to contact their partner themselves (passive referral), have the health provider contact the partner (provider referral), Sits with his/her partner (s) together with trained providers to provide support as they disclose their HIV status while the provider also offers voluntary HTS to the partner (dual referral), or attempt to contact the partner themselves, with the understanding that the health provider would contact the partner should the index client fail to do so (contract referral).

Index clients will be given a choice as to whether or not they want a written referral letter (love letter) to take to their partner.

If the index client agrees, each listed partner will be: (1) contacted, (2) informed that they had been exposed to HIV, and (3) offered voluntary HIV testing services (HTS). 
All index testing must meet $5 \mathrm{Cs}$ and be consensual, confidential, counselling, correct test results, and connected to treatment or prevention services.

\section{Data Sources, Variables, and Collection}

\section{Quantitative}

Data on the index client's (cases) characteristics (age, sex, contacts, ART status), and the contacts' HIV test outcome (yield, initiation status) will be extracted from the HIV index testing registers into a structured pro forma.

\section{Qualitative}

HIV index testing providers will be interviewed by the HIV/TB district medical mentor (trained in both qualitative and quantitative research). It will be an onsite faceto-face interview conducted in English and audio-recorded using an audio-recorder application after obtaining consent. An interview guide will be used to explore the challenges and make suggestions for improving Index contact testing outcomes for HIV.

\section{Data Management and Analysis}

\section{Quantitative}

Data entry and analysis will be performed using the Statistics package for social science software (SPSS version 16.0). Descriptive statistics will be performed to describe the background characteristics of index clients and successful testing of index contacts. The analysis will entail simple frequencies of the main study outcomes and cross-tabulations. The association of index contact testing with the Gender of the index cases will be examined using the ChiSquare test, and unadjusted relative risks (RR) with $95 \%$ confidence intervals (CI) will be calculated.

\section{Qualitative}

A descriptive content analysis by manual coding will be performed to generate categories or themes. Audio-recorded interviews will be transcribed verbatim on the same day by the
HIV/TB district medical mentor. The traditional method of qualitative analysis will be used in the following steps: (a) manual coding; (b) identifying themes, patterns, and relationships; (c) summarizing the data.

After gathering the data, transcribe the source material with a word processor, make multiple photocopies of the text, painstakingly read through and assign codes to the material, cut the pages up into coded passages and then, manually sort the coded text in order to analyze the patterns found. The primary data will be scanned to look for words and phrases commonly used by respondents (word and phrase repetitions). Using the framework for descriptive analysis, responses will be ranged into categories. Recurrent themes will be identified while looking for similarities and differences. The findings of the qualitative analysis will then be linked to the research aim and objectives.

\section{Ethical Considerations}

Informed written consent will be obtained from all key informants before conducting the interviews. Participants are free to skip questions that they may deem personal or otherwise.

Informed written consent will also be obtained from interested and eligible clients before enrolling them as index cases. Since the index testing service that will be offered is part of the recommended national HIV testing services, universal testing principles will be observed for all participants. Study participation will be voluntary. The information collected during the course of this study will be kept confidential. Client's privacy and confidentiality will be observed by assigning a serial number to each participant that will be known only to the health care provider. Only the client's initials and serial number will appear on the data collection forms. Research subjects will also be given an option to opt-out of the project at any time during the implementation period, and this will not affect their access to clinical care they are otherwise entitled to receive. The publication of study findings will report de-identified data. 


\section{Budget}

Table 1. Estimated budget in Zambian Kwacha (ZMW)

\begin{tabular}{|l|l|l|l|}
\hline Item & Unit cost (ZMW) & Number of units & Total cost (ZMW) \\
\hline Tape recorder & 175 & 2 & 350 \\
\hline Cassettes & 15 & $12 \times 2$ & 360 \\
\hline Papers & 80 & 3 & 240 \\
\hline Photocopy & 1 & 3000 & 3,000 \\
\hline Transport refund for community testing & 50 & $6 \times 3 \times 4 \times 6$ & 21,600 \\
\hline Ethical clearance fee & 1,500 & 1 & 1,500 \\
\hline APC & 1,500 & 2 & 3,000 \\
\hline SPSS software & 1,500 & 1 & 1,500 \\
\hline Administration costs & 5,000 & 1 & 5,000 \\
\hline Total & & & $\mathbf{3 6 , 5 5 0}$ \\
\hline
\end{tabular}

\section{Timeframe}

Table 2. Schedule of Activities and Estimated Timeline

\begin{tabular}{|l|l|}
\hline Activity & Time Frame \\
\hline Proposal writing and ethical approval & At the beginning of the study (By December 2019) \\
\hline Collection and analysis of baseline data & 1 to 3 months (December 2019 to February 2020) \\
\hline Design and interview of key informants & By the end of the first month (December 2019) \\
\hline $\begin{array}{l}\text { Analysis of perceived barriers and } \\
\text { design of interventions to address them }\end{array}$ & 1 to 3 months (December 2019 to February 2020) \\
\hline $\begin{array}{l}\text { Application of identified interventions, } \\
\text { including onsite mentorship }\end{array}$ & 1 to 6 months January to June 2020) \\
\hline Data analysis & 1 to 3 months (June to September 2020) \\
\hline Final assessment and report writing & At the end of the project (By December 2020) \\
\hline
\end{tabular}

\section{Glossary of Terms}

AIDS: Acquired Immuno Deficiency Syndrome.

ART: Anti-retroviral Therapy.

Hard to reach populations in HIV programs: middle aged men, adolescent girls and young women.

HIV: Human Immune Deficiency Virus.

HIV testing of the partner was done using determine screening test and confirmed using SD Bioline test after enrollment of the index case. HIV testing is defined as timely if performed within two weeks of the index case enrollment and contacts elicitation, and delayed if done afterwards.

HTS: HIV Testing Services.
Index client or index case: is defined as an individual newly diagnosed as HIV-positive and/or an HIV-positive individual who is newly enrolled in HIV treatment services.

Index contact: is defined as sexual partner or sexual contact of an index case (excluding drug partner and children).

Index testing: voluntary process where counsellors and/or health care workers ask index clients to list all of their sexual partners within the past one year.

LTFU: lost to follow up.

Middle aged men: men between 25 and 40 years.

MOH: Ministry of Health.

Young women: women between 18 and 24 years. 


\section{Results and Discussion}

The expected main findings following the address of the identified challenges are: increased number of recipients of care indexed, increased index contact elicitation ration from $1: 1$ to at least $1: 2$, improved rate of contacted tested on time, improved HIV case finding through index testing and overall, increased HIV index testing positivity rate from below $25 \%$ (baseline) to above $40 \%$ (at the end of the project) $[14,15]$.

Barriers Identified might include the following:

1. Provider related challenges such as Inadequate elicitation skills and knowledge gaps among providers; and some logistic challenges.

2. Clients related challenges such as client mobility and migration; and lack of accurate information for client and contact location.

Interventions will be designed and implemented according to barriers identified to improve quality of index testing services provided.

In this study, the Pearson Chi-square analysis will be used to ascertain the statistical significance of the difference between gender for new HIV positive clients identified.

\section{References}

[1] UNAIDS, Joint United Nations Programme on HIV/AIDS, Global AIDS Update 2018. Miles to go: Closing Gaps; Breaking Barriers; righting injustices. https://www.unaids.org/en/resources/documents/201 8/global-aids-update.

[2] Zambia Population-Based HIV Impact Assessment Zamphia 2015-2016.

https://phia.icap.columbia.edu/wp-

content/uploads/ZAMBIA-Factsheet.FIN_.pdf.

[3] AVERT, Global information and education on HIV and AIDS, HIV and AIDS in Zambia, 2018. https://www.avert.org/professionals/hiv-aroundworld/sub-saharan-africa/zambia.

\section{Conclusion}

Following appropriate interventions (enhanced partner notification) aiming at addressing barriers to index testing in Lusaka, the HIV positivity yield will increase from below $25 \%$ at baseline to above $40 \%$ at the end of the study. HIV programs will then need to explore and address barriers to HIV partner testing services to maximize HIV case identification.

\section{Disclosure}

The author reports no conflicts of interest in this work.

\section{Acknowledgements}

I am highly appreciative of the CDC for the opportunity given to me to serve as Medical Mentor in the CDC/LPHO cooperative agreement and mentorship program. I am also thankful to the Lusaka Provincial Health Office for availing me with the unforgettable and lifechanging opportunity which saw me lead the HIV/TB project in Matero Sub-district of Lusaka, Zambia.

My most honest and sincere gratitude goes to Dr. Monde Muyoyeta for taking me through the process of research protocol development and for her supervisory support throughout the thesis.

[4] M. Katbi, A. Adegboye, A. Adedoyin, F. Yunusa, et al. Effect of clients Strategic Index Case Testing on community-based detection of HIV infections (STRICT study).

https://www.ncbi.nlm.nih.gov/pubmed/term=Effect+ of+clients+Strategic+Index+Case+Testing+on+com munity-

based+detection+of+HIV+infections+(STRICT+stu dy).

[5] Partner and Family based Index case testing. A Standard Operating Procedure (SOP). https://static1.squarespace.com/static/5a29b53af9a6 1e9d04a1cb10/t/5bcf3f4324a69482bb98d34f/15403 08814679/Index+Testing+SOP+slides+_v12+Octob er+10+2018.pdf. 
[6] Marya Plotkin; Catherine Kahabuka; Alice Christensen; Denice Ochola. Outcomes and Experiences of Men and Women with Partner Notification for HIV Testing in Tanzania: Results from a Mixed Method Study. Open access publication, published online on 31 October 2017. https://www.ncbi.nlm.nih.gov/pmc/articles/PMC575 $8678 /$.

[7] Gari S, Malungo JRS, Martin-Hilber A, Musheke M, Schindler C, Merten S. HIV testing and tolerance to gender based violence: a cross-sectional study in Zambia.

https://www.ncbi.nlm.nih.gov/pmc/articles/PMC374 9220/.

[8] World Health Organization. HIV status disclosure to sexual partners: rates, barriers, and outcomes for women. Geneva; 2003.

http://apps.who.int/iris/bitstream/10665/42717/1/924 1590734.pdf.

[9] Obermeyer C, Osborn M. The utilization of testing and counseling for HIV: a review of the social and behavioural evidence.

https://www.ncbi.nlm.nih.gov/pmc/articles/PMC199 $4175 /$.

[10] K. Selvaraj, A. M. V. Kumar, S. Chawla, K. S. Shringarpure, et al. Are partners of HIV-infected people being tested for HIV? A mixed-methods research from Gujarat, India. https://www.ncbi.nlm.nih.gov/pmc/articles/PMC552 6482/.

[11] Skovdal M, Campbell C, Madanhire C, Mupambireyi Z, Nyamukapa C, Gregson S. Masculinity as a barrier to men's use of HIV services in Zimbabwe. Global Health. 2011; 7:13. Published 2011 May 15. doi:10.1186/1744-8603-7-13 https://www.ncbi.nlm.nih.gov/pmc/articles/PMC310 $7786 /$.

[12] Obermeyer CM, Osborn M. The utilization of testing and counseling for HIV: a review of the social and behavioral evidence. Am J Public Health. 2007;97(10):1762-1774.

doi:10.2105/AJPH.2006.096263

https://www.ncbi.nlm.nih.gov/pmc/articles/PMC199 $4175 /$.

[13] Obermeyer CM, Sankara A, Bastien V, Parsons M. Gender and HIV testing in Burkina Faso: an exploratory study. Soc Sci Med. 2009;69(6):877-884. doi:10.1016/j.socscimed.2009.07.003 https://www.ncbi.nlm.nih.gov/pmc/articles/PMC426 0152/.

[14] Brown LB, Miller WC, Kamanga G, et al. HIV partner notification is effective and feasible in subSaharan Africa: opportunities for HIV treatment and prevention. $J$ Acquir Immune Defic Syndr. 2011;56(5):437-442.

doi:10.1097/qai.0b013e318202bf7d

https://www.ncbi.nlm.nih.gov/pmc/articles/PMC320 $7356 /$.

[15] Plotkin M, Kahabuka C, Christensen A, et al. Outcomes and Experiences of Men and Women with Partner Notification for HIV Testing in Tanzania: Results from a Mixed Method Study. AIDS Behav. 2018;22(1):102-116. doi:10.1007/s10461-017-1936$\mathrm{X}$ https://www.ncbi.nlm.nih.gov/pmc/articles/PMC575 $8678 /$. 